\title{
Telephone versus in-person intake assessment for bereavement intervention: Does efficiency come at a cost?
}

\section{Catherine Newsom, Henk Schut, Margaret Stroebe, John Birrell \& Stewart Wilson}

To cite this article: Catherine Newsom, Henk Schut, Margaret Stroebe, John Birrell \& Stewart Wilson (2016) Telephone versus in-person intake assessment for bereavement intervention: Does efficiency come at a cost?, Death Studies, 40:2, 71-79, DOI: 10.1080/07481187.2015.1068244

To link to this article: http://dx.doi.org/10.1080/07481187.2015.1068244

Accepted author version posted online: 30 Nov 2015.

Published online: 30 Nov 2015.

Submit your article to this journal $₫$

Џ Article views: 25

Q View related articles $\longleftarrow$

View Crossmark data $\nearrow$ 


\title{
Telephone versus in-person intake assessment for bereavement intervention: Does efficiency come at a cost?
}

\author{
Catherine Newsom ${ }^{\mathrm{a}, \mathrm{b}}$, Henk Schut ${ }^{\mathrm{a}}$, Margaret Stroebe ${ }^{\mathrm{a}, \mathrm{c}}$, John Birrell ${ }^{\mathrm{b}}$, and Stewart Wilson ${ }^{\mathrm{b}}$ \\ aDepartment of Clinical Psychology, Utrecht University, Utrecht, The Netherlands; ${ }^{\text {b} C r u s e ~ B e r e a v e m e n t ~ C a r e ~ S c o t l a n d, ~ P e r t h, ~ S c o t l a n d ; ~}$ \\ 'Department of Clinical Psychology and Experimental Psychopathology, University of Groningen, Groningen, The Netherlands
}

\begin{abstract}
Standardized, evidence-based risk assessment is an important component in providing effective bereavement care. E-health intake assessments have been offered alongside or instead of in-person assessments, although evidence concerning the equivalence of assessment results is lacking. This article examines differences between a semistructured intake assessment for grief intervention conducted over the telephone $(n=330)$ and in-person $(n=115)$. Differences in scores and clinical implications were evaluated. Although composite assessment scores were lower in the telephone condition, further examination revealed this occurred in the semistructured assessment of risk of complications, not the structured grief symptom assessment. Implications for care provision are discussed.
\end{abstract}

Research has indicated that bereaved people whofor various reasons-are considered to be at risk of experiencing complications in the grieving process are the ones on whom professional bereavement interventions should focus (Currier, Holland, \& Neimeyer, 2006; Schut \& Stroebe, 2005). This calls for valid assessment of grieving difficulties to identify such vulnerable people because standardized, evidence-based risk assessment is an important component in the provision of effective bereavement care (Schut \& Stroebe, 2005). Such assessment enables care providers to offer the right kind of help to those who need it, and-of equal importance - to avoid providing intervention to people who will probably not benefit from it. In addition, psychotherapists and counselors are increasingly under pressure to operate as efficiently as possible while minimizing operating costs. In this context, offering intake assessment over the telephone, by video conference call, or by means of other e-health technologies has been considered a potential way of reducing costs for clients and practitioners in recent years, increasing availability to serve people needing help, and identifying which people stand a good chance of benefiting from intervention and which individuals will most likely cope better on their own.

Drawing from the broader e-health literature, remotely-conducted intake assessment offers unique advantages and disadvantages (Crawford, 2003; Freir et al., 1999; O’Reilly et al., 2007). Potential advantages include cost savings, physical and social accessibility, and the extent of available intervention services (see Lee et al., 2010; Peñate, 2012). Another possible advantage is the reduction in fear of social stigma associated with accessing psychological or psychiatric services (see Cukor et al., 1998; Kavanagh \& Yellowlees, 1995; Peñate, 2012). Doubts about such assessment have to do with the quality of the encounter (e.g., commitment regarding keeping appointments, and the active engagement of the client; see Mozer, Franklin, \& Rose, 2008). Indeed, O'Reilly et al. (2007) observed that $10 \%$ of people approached to participate in a videoconferencing study declined to do so, citing their unwillingness to receive services remotely. Concerning the broader range of e-health modalities, access to and familiarity with technological platforms must also be considered. Modalities can range from a basic telephone call to low-cost internet telephony applications for audio and video conference calls to social media or virtual reality programs (see Peñate, 2012). At the time of writing, factors such as advanced age, lower education level, and lower socioeconomic status were liable to restrict access.

In-person assessment modalities have their own advantages and disadvantages. The advantages range from feasibility to a preference for seeing a counselor in person (see Cukor et al., 1998). Indeed, some people cannot or do not wish to use telephones or other communications technology, whereas others may not have 
adequate privacy when they use such devices to answer personal questions. In their meta-analysis of remote assessments and interventions, García-Lizana and Muñoz-Mayorga (2010) concluded that a preference for in-person over remote assessment predominates among both clients and practitioners. The disadvantages to in-person assessment mirror some of the advantages of remote assessment; for example, the relatively higher costs of in-person services and the potential physical and social (e.g., stigma) barriers to accessing care at a counseling location.

Given the range of potential advantages and disadvantages, the impact of integrating an e-health assessment procedure into a bereavement intervention program needs careful consideration. There are various ways that evaluation of e-health initiatives can be approached. Guidelines recommended by The American National Institutes of Health for evaluating telehealth initiatives follow Hicks et al.'s (2011) transactional economics-based model, which stipulates concentrating on one of three foci of analysis (cost, quality, or access) and one of three levels of analysis (society, community, or individual). To date, no evaluations of e-health versus in-person assessments have been conducted for bereavement intervention programs. Although a great deal of research has been conducted recently on the efficacy of e-health interventions for a range of psychological health conditions and therapies among different populations, our focus is on assessment in a telehealth context. Specifically, the present study is interested in the quality of an assessment for bereavement intervention (the similarity of results across conditions) at the individual level (the assessment of bereaved persons' grieving difficulties).

The study was designed, first, to compare the results of assessment for the two modes of intake: Is there a difference in evaluation of the client's difficulties in the grieving process (including level of grief symptomatology and risk of grief complications) when assessed remotely (telephone intake) or in person? Second, the clinical implications of using one or the other mode of assessment were explored: Are there differences in assignment to specific types of bereavement intervention depending on whether remote or in-person intake has been used? In the present study, the telephone was selected as the means for delivering the remote assessment for several reasons, which are discussed below.

Given that research on intake procedures specific to assessment for bereavement intervention is lacking, scientific literature from a wider range of telephonebased psychological and psychiatric assessment services is briefly reviewed in the next section. The rationale and design of the present study are then outlined.

\section{Telephone versus in-person assessment: Review of empirical studies}

In general, it has been shown that remote and in-person assessments have produced similar results when it comes to practitioners' assessment of clients' mental health, particularly when structured interviews are used (for social anxiety disorder, see Crippa et al., 2008; Lyneham \& Rapee, 2005; for depression, see Pinto-Meza, SerranoBlanco, Peñarrubia, Blanco, \& Haro, 2005; Simon, Revicki, \& Von Korff, 1999; Spek, Nyklíček, Cujpers, \& Pop, 2008; for Axis I and II disorders, see Brar et al., 2002; Rohde, Lewinsohn, \& Seeley, 1997; for a metaanalysis of studies, see Hyler, Gangure, \& Batchelder, 2005). Exceptions have also been reported, indicating the need for careful consideration, interviewer training, and clearly established and communicated guidelines before implementing a telephone-based assessment program where questions may address sensitive personal matters. Although Rohde et al. (1997) reported generally quite equivalent results from telephone and in-person interviews, significant discrepancy was noted in the telephone condition participants' responses to substance abuse questions. The authors suggested that this may have been attributable to lack of privacy, as several participants attended telephone interviews with their children or spouses in the room.

Investigations of the equivalence of semistructured diagnostic assessments conducted over the telephone and in person, however, have produced mixed results. In a study using a test-retest design to examine the equivalence of telephone and in-person delivery of the Structured Clinical Interview for DSM-IV for Axis I disorders (SCID I), Cacciola, Alterman, Rutherford, McKay, and May (1999) reported a significantly higher number of diagnoses of lifetime major depression in the in-person interviews than in the telephone interviews. They also found poor agreement with social phobia. The authors suggested that the in-person bias in the diagnosis of major depression may have been attributable to the opportunity-introduced by the semistructured interview format-for the clinical interviewers to make use of their professional skills to gather information. Given that they were not specially trained for assessment via telephone, the interviewing clinicians in the study may have been better able to probe for further information in the in-person assessment format to which they were accustomed, and less adept at eliciting further information over the telephone. Crippa et al. (2008) repeated Cacciola et al.'s investigation using the SCID-I for social anxiety with results that demonstrated equivalence across telephone and in-person conditions. They attributed the difference in results from Cacciola 
et al.'s study to the extensive training process that their interviewers went through to prepare for telephone delivery, including role-plays and simulations, discussion of potential responses and their ratings, and observation of interviews.

Based on the general findings reported above, there seem to be good reasons to expect that telephonedelivered and in-person assessment can yield equivalent results in terms of quality in a structured format, and possibly also in a semistructured format when sufficient interviewer training is provided.

\section{Telephone versus in-person assessment for bereavement-related grief symptoms and risk}

Existing reservations about the quality of telephone versus in-person assessment of the psychological services identified above also imply the need for specific quality assessment of these assessment modalities in the bereavement intervention area. In other words, we need to determine whether it is appropriate to deliver an assessment of bereavement-related grief symptoms and risk remotely or in person, and whether assessment results from the two delivery methods can be integrated into the same client intake system. Furthermore, it is necessary to understand whether the assessment results differ, and if so, in what way.

For that purpose, a naturalistic quasi-experimental study was conducted, first, to compare the results of assessment for the two modes of intake: Are there differences in evaluation of the client's grief complication risks when assessed over the telephone or in person? Second, if the previous question was to be confirmed, the clinical implications of using one or the other mode of assessment needs be explored: Are there differences in assignment to specific types of bereavement intervention depending on whether telephone or in-person intake has been used? Given the lack of grief-specific evidence in the reviewed literature, the current study was exploratory in nature.

\section{Method}

The opportunity to conduct this naturalistic investigation was presented in the context of a larger study on the efficacy of the national bereavement care service implemented by Cruse Bereavement Care Scotland (CBCS), a charity that provides over 12,000 hours of one-to-one bereavement support and serves over 3,000 bereaved people each year. Because of long waiting lists for bereavement intervention services and the organization's broad geographical remit, CBCS introduced telephone-based assessment in 2011 to expand its overall capacity for assessment. CBCS considers evaluation of this extension to the care program an important step in its implementation. The telephone was selected as the means of remote assessment delivery (rather than video conferencing or other web-based technologies) by the service-providing organization because it remains the most accessible means of telecommunication for most of the UK population. As of May 2013, 14\% of the UK population aged 75 and over had never used the Internet. Scotland also continues to struggle with poor quality Internet connections across its regions, although the government has set an objective to develop a high-quality Internet infrastructure across Scotland by 2020 (Scottish Government, 2011).

\section{Participants}

Potential participants were identified (with client consent) via the CBCS database. All participants had initiated contact with the bereavement support organization themselves and had actively requested support. All were adults (age 18 and over) who had been bereaved for at least 6 months prior to the intake assessment. For inclusion in the study, no distinction was made with respect to the participants' relationship to the deceased, which nevertheless followed a similar pattern in both conditions and was representative of general CBCS client demographics. A description of participants' demographic information can be seen in Table 1 .

Because testing for equivalence is testing the null hypothesis, the issue of sample size is important. To estimate an appropriate sample size, we have assumed that a difference of one-half of a standard deviation

Table 1. Study participant details by condition.

\begin{tabular}{|c|c|c|c|c|c|}
\hline & \multicolumn{2}{|c|}{ In-person } & \multicolumn{2}{|c|}{ Telehealth } & \multirow{2}{*}{$\begin{array}{c}\text { Total } \\
\mathrm{n}= \\
445\end{array}$} \\
\hline & $\begin{array}{l}\mathrm{n}= \\
330\end{array}$ & $100 \%$ & $\begin{array}{l}\mathrm{n}= \\
115\end{array}$ & $100 \%$ & \\
\hline \multicolumn{6}{|c|}{ Relationship to the deceased Participant was: } \\
\hline Parent of an adult child & 43 & 13 & 16 & 14 & 59 \\
\hline Parent of a child under 18 & 10 & 3 & 3 & 3 & 13 \\
\hline Partner & 88 & 26 & 43 & 37 & 131 \\
\hline Adult child of a parent & 126 & 38 & 41 & 36 & 167 \\
\hline Sibling & 35 & 11 & 7 & 6 & 42 \\
\hline Other & 28 & 9 & 5 & 4 & 33 \\
\hline \multicolumn{6}{|l|}{ Cause of death } \\
\hline Health problem & 189 & 57 & 62 & 54 & 251 \\
\hline Accident & 23 & 7 & 11 & 10 & 34 \\
\hline $\begin{array}{l}\text { Other (murder, suicide, drug } \\
\text { or alcohol - related) }\end{array}$ & 109 & 33 & 36 & 31 & 145 \\
\hline Missing / not available & 9 & 3 & 6 & 5 & 15 \\
\hline \multicolumn{6}{|l|}{ Time since bereavement } \\
\hline Between 6 months and 1 year & 148 & 45 & 59 & 51 & 207 \\
\hline Between 1 and 2 years & 82 & 25 & 29 & 25 & 111 \\
\hline Between 2 and 3 years & 33 & 10 & 8 & 7 & 41 \\
\hline Between 3 and 5 years & 23 & 7 & 10 & 9 & 33 \\
\hline Over 5 years & 42 & 13 & 9 & 8 & 51 \\
\hline Not available/missing & 2 & $<1$ & 0 & 0 & 2 \\
\hline
\end{tabular}


between the two groups would be sufficiently large to suggest nonequivalence. An apriori power analysis indicated that a sample of 210 participants (105 per group) would have a $95 \%$ chance of detecting the effect if the population difference were at least one-half of a standard deviation.

In total, data from 445 participants were selected for the study, with 115 in the telephone condition, and 330 in the in-person condition (eight participants had to be excluded from the study for various reasons; e.g., a few clients presented with substance abuse problems that required attention before bereavement intervention could be considered).

Clients in both conditions were contacted via telephone by a scheduling administrator. Assignment to modality was determined by participants' position on the CBCS waiting list, which operates on a first come, first serve basis. This quasi-randomization worked as follows: Clients contacted for scheduling on certain days were offered telephone interviews to be scheduled at a convenient time, whereas those contacted on other days were offered in-person appointments. A limited number of potential participants declined telephone interviews for a variety of reasons, including hearing issues and discomfort speaking over the phone.

It is important to note that although previous epidemiological research has indicated that people with anxiety and depressive disorders may be more likely to turn down interviews out of reluctance to be evaluated (Eaton et al., 1992) and might therefore prefer telephone interviews to in-person ones, the context of our naturalistic study makes it unlikely for severity of grief-related depression symptoms to have affected participation rates in the telephone condition any more or less than in the in-person condition. Participants who had opted out of the telephone condition were excluded from the study (but not from assessment or potential intervention). An additional number of participants opted out of the in-person assessment interview as well, but this number cannot be differentiated from clients who changed their minds and chose not to pursue support altogether.

The participants in the two conditions did not differ in terms of relationship to the deceased, $\chi^{2}(4, n=445)$ $=8.22, p=.084$; cause of death, $\chi^{2}(2, n=430)=.96$, $p=.619$; gender, $\chi^{2}(1, n=441)=.22, p=.643$; or time since bereavement, measured as a scale variable, $\chi^{2}(5$, $n=443$ ) $=6.48, p=.263$ (numbers differ slightly from the total study participation number as data for certain variables were missing in a few cases). Given that data on participants' age were collected in age ranges according to standard procedure at the counseling organization, another chi-square test was conducted to determine differences between categories in terms of participants' ages. The results indicated a small but significant association between age and category, $\chi^{2}(5, n$ $=411)=14.89, p=.011$, Cramer's $\mathrm{V}=.19$. A Bonferroni-corrected Z-test of proportions revealed a significant difference in two age categories. A significantly greater proportion of those in the face-to-face condition were $18-29$ years of age $(14 \%)$ compared to the proportion of those in the phone-based condition $(4 \%)$. In contrast, a significantly greater proportion of those in the phone-based condition were aged 60-74 (28\%) as opposed to those in the face-to-face condition $(15 \%)$. These group differences are presented in detail in Table 1. We explored whether the difference in age had any impact on the dependent variable (assessment results) using multiple regression analysis and found no significant effect for age, as detailed below in the Results section.

\section{Instruments}

The assessment instrument used was the Indicator of Bereavement Adaptation-Cruse Scotland (IBACS). The IBACS was specifically designed to identify the level of risk and complexity of grief symptoms presented by the bereaved person in order to provide a commensurate level of bereavement support. The IBACS total score is calculated by the interviewer directly at the conclusion of the session. The IBACS is comprised of two sections:

\section{Risk assessment}

A semistructured interview was conducted addressing a number of risk factors that have been shown to contribute to complicated or prolonged grief (cf. Stroebe, Folkman, Hansson, \& Schut, 2006; Zisook et al., 2014). These include the client's personal and health history (including previous bereavements, losses, and traumas; personal health problems; and past psychological problems), relationship to the deceased (e.g., if the bereaved person has lost a child or if the relationship was complicated), social support (if the bereaved person was extremely lonely), and additional stressors (employment problems, caregiving responsibilities, financial problems, relocating) considered to contribute to complications in the grieving process. The risk assessment can produce a subtotal of 0 (standard) to a maximum of 7 points. Interviewers are instructed to provide no more than 1 point per category of risk.

\section{Intensity of grief}

This part was comprised of a 12-item self-report questionnaire primarily developed with permission from the Inventory of Complicated Grief-revised (Prigerson 
et al., 1995; Prigerson \& Jacobs, 2001). Items were selected for a Scottish clientele and integrated to suit the Scottish context. Items on the questionnaire were rated on a $0-4$ point Likert scale. Two specific items addressed client suicidal ideation. These items contributed to the total result but were also tabulated separately to indicate the client's risk of suicide. Internal consistency of this section of the IBACS was assessed using a separate client sample that included data on each individual item. This sample $(n=331)$ showed acceptable internal consistency of the instrument, with a Cronbach's alpha of 75 .

The IBACS total score consists of the sum of the 12 grief symptomatology items from the self-report, along with $0-7$ additional discretionary risk assessment points allocated by the interviewer based on the client's responses to the Part 1 risk assessment questions. A client's IBACS point total can range from 0 to 55 . Cruse Bereavement Care Scotland developed a tiered model whereby these points can be categorized into groups to enable clients to be assigned to an appropriate level of care $(0-17=$ no intervention or watchful waiting; $18-$ $28=$ skilled listener; $29-38=$ advanced skills listener; $39-55=$ counselor). An exception is made when clients report a high score on the two suicidality questions (a cumulative score of 6 points or higher), which make up part of the 12-item self-report. These two suicidality items are included in the IBACS total but are also considered as an independent subscale for suicide risk. In cases where a high suicide risk is reported, in-person and telephone interviewers alike are instructed to follow the CBCS suicide-risk protocol, which is designed to avail clients of appropriate and immediate support. This did not occur in the present study sample.

In the current sample, complete IBACS assessment forms were available for 125 participants, allowing for a comparison of risk assessment and grief scores across conditions. For the remaining 320 participants, only the IBACS point total was available for analysis.

\section{Procedure}

All interviewers completed a bereavement-specific assessment training module provided by CBCS. The specific training for the IBACS role includes an e-learning module on the place of assessment in a tiered intervention model and an understanding of data protection and interview skills. Trainees are invited to think about their script for explaining these issues to a prospective client. Successful completion of the e-learning module is followed by 2 days of group training. On Day 1, the delivery of the assessment interview is discussed in detail and skills are practiced and peer assessed. Following Day
1, trainees are provisionally licensed to conduct three IBACS sessions, which they then submit for evaluation. Trainees present details of these sessions on Day 2 of the training. A discussion of issues raised is followed by a discussion around discretionary points and ethical issues, such as supervision of IBACS sessions conducted by telephone. At the time of data collection, specific training for telephone delivery of the IBACS consisted of approximately $3 \mathrm{hr}$ of additional discussion and practice work.

All interviews were conducted between December 2010 and April 2011. The same group of experienced interviewers conducted interviews in both modalities. Because at the time of data collection only this group of CBCS interviewers had conducted the IBACS both over the telephone in the pilot study and in person, eligibility for inclusion in the scientific study was further restricted to those clients who had been interviewed by one of those interviewers. This inclusion criterion was intended to reduce differences between the telephone and in-person conditions by controlling for differences between individual interviewers.

\section{Results}

The first objective was to establish whether there were differences in participants' IBACS point totals (the composite level of grief symptomatology and risk assessment score) according to in-person or telephone modalities. An independent samples $t$ test was conducted on the IBACS point total and revealed a significant difference (with a medium effect size) between the telephone and in-person conditions, $t(442)=2.96, p=.003, d=.33$. Participants in the telephone condition had significantly lower scores $(n=112, M=26.05, S D=8.24)$ than participants in the in-person condition $(N=332, M=28.76$, $S D=8.42$ ).

Implications for assignment of clients to intervention categories based on the CBCS model were then considered. Further examination of the mean differences between the in-person and telephone conditions revealed that a client with an average in-person assessment total (29 points) would be assigned to the advanced skills listener intervention category, whereas a mean score in the telephone condition (26 points) would indicate assignment to the skilled listener category.

However, as noted earlier, there was a slight difference in participants' ages between the two delivery methods. We therefore conducted a linear regression predicting total IBACS points, controlling for participant age group. This analysis was important to dismiss the possibility that differences in IBACS points between 
the two methods were due only to age differences between the delivery methods. The overall model predicting IBACS total with age group (dummy coded) and IBACS delivery method entered as predictors was significant, $F(6,401)=6.25, p<.001, R^{2}=.086$. After controlling for the effects of age, the IBACS delivery method was a significant predictor of total IBACS points, $t(401)=2.96, p=.003$. Using the predicted IBACS total points generated by the regression that was adjusted for age, participants in the telephone condition had an average IBACS point total of 25.82 $(n=96, S D=2.14)$, and participants in the in-person condition had an average IBACS point total of 28.73 $(n=312, S D=2.16)$. The results indicate that when controlling for age, the difference between point totals for the two delivery methods $\left(M_{\mathrm{dif}}=2.86, \mathrm{SE}=.97\right)$ was very similar to the difference between delivery methods when not controlling for age $\left(M_{\text {dif }}=2.92\right.$, $S E=.98)$. These differences are based on the unstandardized betas using the same sample of 408 participants for whom age was available.

To further assess this point, the resulting assignment to the intervention category for participants-based on IBACS cutoff points-was compared according to method of delivery. A chi-square test for independence indicated no statistically significant association between IBACS delivery method and assignment to specific intervention category, $\chi^{2}(3, n=447)=6.34, p=096$, Cramer's $V=.12$. Table 2 presents the percentage of assignments in this sample to each intervention category (including no intervention) by IBACS delivery method. The proportion of IBACS interviewees in the telephone category who were found not to require intervention was greater than the proportion of participants in the in-person condition $(13 \%$ telephone, $8 \%$ in person). Although this effect was not statistically significant, the results illustrate a trend where those who received telephone assessment were more likely to be assigned to receive less complex intervention.

Although the mean difference between the conditions was not statistically significant, it did translate to a clinical difference in the IBACS system of cutoff points. It was therefore considered important to investigate the mechanism underlying the difference in means between the telephone and in-person conditions. First, a simple

Table 2. Intervention category assignments ( $\%)$.

\begin{tabular}{lcc}
\hline Intervention category & In-person (\%) & Telehealth (\%) \\
\hline No intervention & 8 & 13 \\
Skilled Listener & 41 & 50 \\
Advanced Skills Listener & 37 & 28 \\
Counsellor & 14 & 9 \\
Total & 100 & 100 \\
\hline
\end{tabular}

linear regression model was conducted predicting IBACS points from method of delivery to determine whether the difference between the in-person and telephone group might be due to demographic and loss factors. This model was then compared to a multiple linear regression model which predicted IBACS points from method of delivery, client's age, length of bereavement, relationship to the deceased, and the deceased person's cause of death. A reduction in the magnitude of the effect for in-person versus telephone conditions would reveal whether the demographic and loss factors accounted for the difference in IBACS points between these two conditions. The analysis was conducted via a hierarchical regression model with method of delivery entered in block 1 and the other demographic and loss variables added in block 2 . Both block 1 and block 2 were significant $(N=395, p<.01)$. Using the predicted IBACS total points generated by the regression that was adjusted for the demographic and loss variables (including age), participants in the telephone condition had an average IBACS point total of $25.62(n=93$, $S D=3.06)$, and participants in the in-person condition had an average IBACS point total of $28.60(n=302$, $S D=2.84$ ). The results indicate that when controlling for the demographic and loss variables-similar to when controlling for only age-the difference between point totals for the two delivery methods $\left(M_{\mathrm{dif}}=2.91\right.$, $S E=.98)$ remained very similar to the difference between delivery methods when not controlling for any variables $\left(M_{\mathrm{dif}}=3.00, \mathrm{SE}=.99\right)$. These differences are based on the unstandardized betas from Block 1 and Block 2 using the same sample of 395 participants for whom the demographic and loss variables were available. When comparing the unstandardized betas from Block 1 and Block 2, the difference is not significant $(p=.948)$. In summary, although the demographic and loss factors together did significantly predict IBACS points in this model $(p<.001)$, they did not account for the difference between the telephone and in-person delivery methods.

\section{Risk assessment points}

As noted above, data on the breakdown of total IBACS points into risk assessment points and intensity of grief points were available only for a subset of participants ( $n=124 ; 81$ in person, 43 telephone). The subsample had a similar demographic composition to the larger sample-including a mixture of urban, suburban, and rural locations-and as such was used to provide insight into whether the difference in total IBACS points between the two delivery methods may be attributed to differences in the risk assessment points, differences in 
the intensity of grief scores, or both when the size of the effect is large enough. When the IBACS sum scores within this subset of the data were analyzed, the difference in total IBACS points between conditions was not statistically significant (in person, $n=81, M=29.20$, $S D=9.13$; telephone, $n=43, M=27.19, S D$ 7.18), $t(122)=1.25, p=.213, d=.24$. The post hoc effect size of the $t$ test was revealed to be only .41, indicating that the test was underpowered and the sample size may have been inadequate to show a significant difference. However, because the difference between the in-person and telephone conditions in the subsample was comparable to the difference found in the full sample, we nevertheless investigated whether this more modest group difference was evident in the risk assessment portion or the grief intensity portion of the assessment tool.

A $t$ test was conducted to determine whether the inperson and telephone conditions differed significantly on the risk assessment portion of the IBACS. Because the variances of the two groups differed significantly, a $t$ test for nonequivalent variances was used. This test indicated that participants who received an in-person assessment were assigned more risk assessment points $(M=3.16, S D=2.04)$ than were participants who received a telephone assessment $(M=1.23, S D=1.49$; $t(112)=6.05, p<.001)$. This finding was confirmed with a nonparametric Mann-Whitney test $(Z=5.00$, $p<.001$ ), which we conducted due to slight violations in the normality of the risk assessment point total.

To establish whether the difference between the in-person and telephone conditions in the number of risk assessment points may be due to demographic and loss factors, a simple linear regression model was conducted predicting risk assessment IBACS points from method of delivery. This model was then compared to a multiple linear regression model which predicted IBACS risk assessment points from method of delivery, client's age, length of bereavement, relationship to the deceased, and the deceased person's cause of death. A reduction in the magnitude of the effect for in-person versus telephone condition would reveal whether the demographic and loss factors account for the difference in IBACS points between the telephone and in-person conditions. The analysis was conducted via a hierarchical regression model with method of delivery entered in Block 1 $\left(n=114, F(1,112)=28.95, p<.001, R^{2}=.205\right)$ and the other demographic and loss variables added in Block 2 $\left(n=114, F(16,97)=3.52, p<.001, R^{2}=.367\right)$. Using the predicted IBACS total points generated by the regression that was adjusted for the demographic and loss variables (including age), participants in the telephone condition had an average IBACS point total of $1.25(n=40, S D=1.06)$, and participants in the in-person condition had an average IBACS point total of $3.20(n=74, S D=.82)$. The results indicate that when controlling for the demographic and loss variables, the difference between point totals for the two delivery methods $\left(M_{\mathrm{dif}}=1.81, S E=.38\right)$ was slightly smaller than the difference between delivery methods when not controlling for any variables $\left(M_{\mathrm{dif}}=1.98, S E=.37\right)$. These differences are based on the unstandardized betas from Block 1 and Block 2 using the same sample of 114 participants for whom the demographic and loss variables were available. When comparing the unstandardized betas from Block 1 and Block 2, the difference was not significant $(p=.749)$. In summary, although the demographic and loss factors together did significantly predict risk assessment points $(p=.047)$, they did not account for the difference between the telephone and in-person delivery methods.

A comparable $t$ test was conducted to determine whether the in-person and telephone groups differed on the grief intensity portion of the IBACS (the 12-item grief symptom self-report, which comprises Part 2 of the IBACS). This $t$ test indicated no significant difference between groups, $t(122)=.01, p=.99$.

To summarize the overall pattern of results, the IBACS sum score for participants in the in-person assessment category was on average 3 points higher than the sum score of participants in the telephone assessment category. In the subset of the study for which a breakdown of the IBACS score was available, interviewers assigned participants in the in-person IBACS assessment category on average nearly 2 risk points more than they assigned participants in the telephone-based IBACS assessment category. In contrast, no statistically significant difference was found between the two conditions' grief symptom self-report subtotals. The differences in IBACS sum scores and risk assessment points between the two delivery methods were not significantly affected by controlling for demographic and loss factors. In sum, the assessment of clients' risk of developing complications in grief is higher when the interview is conducted in person, and lower when it is conducted by telephone.

\section{Discussion}

The results of our analyses demonstrate a difference for intake modality on assessment for bereavement intervention. In the subset of the data where a breakdown of the total score was available (including specific information on risk points), it was demonstrated that the variation in point allocation based on risk assessment could be uniquely predicted by intake interview delivery method. This difference was not accounted for by demographic and loss-related variables or by the grief symptom 
subscale scores (i.e., the structured question set). Clients who had in-person intake assessments received more IBACS risk points than clients who had their intake assessments by telephone, but the assignment to intervention categories was shown not to differ despite the significant difference in group means. We can cautiously conclude (while encouraging further investigation) that telephone assessment produces similar results, at least regarding grief symptom level evaluation. These results fit with the broader mental health assessment literature, and we can thus conclude that telephone-based interviews can be integrated into bereavement intervention intake assessment procedures with proper training.

First, we can only speculate as to why participants in the telephone condition received fewer risk points than those in the in-person condition. It is possible that clients assessed remotely were less willing to report risk factors, or that clients assessed in person were more emphatic in their risk reporting. Rohde et al. (1997) found that participants underreported substance abuse symptoms-which, as they noted, stem from socially stigmatizing behaviors-over the telephone. In Scotland, as in other countries with a strong Calvinist legacy, the expression of symptoms of grief and difficulties with coping may not comply with the social ideal of personal strength and unflappability; nevertheless, it is unlikely that it would carry the same degree of stigma that (illegal) substance abuse might. In addition, because results in the remote and in-person conditions were similar in the second part of the intake assessment, it seems improbable that remote clients would be as forthcoming as in-person clients in reporting grief symptoms but more restrictive when it came to risk information.

Second, it may be that important cues-visual or otherwise-indicating risk were not perceived by the interviewers during telephone assessments or were considered to be of greater importance during in-person assessments. However, that appears unlikely to be the case. Baigent et al. (1997) reported differences between clinical interviewers' assessments of blunting of affect and other observed behaviors when they were participating in an assessment remotely (via videoconference) compared to in person. Although all observing clinical assessors had the same visual cues, those assessors who were conducting the assessment remotely rated blunting of affect lower than did the on-site assessors. For this reason, it would appear that absence of visual data itself might not be responsible.

Third, the specific effects of physical presence in the in-person assessment and the kinds of suprasegmental information that may be gained or lost in either scenario should be considered. In particular, it may be worth examining how physical presence affects the transfer of such information, which may also be viewed in terms of the rapport or empathy established between the interviewer and the bereaved client. Depending on the client's expectations and circumstances, this kind of communication may be facilitated more effectively by means of a telephone or in-person encounter. Better understanding of the kind of suprasegmental information that is shared and how it affects the development of rapport between an interviewer and a client in an assessment context would be valuable for improving standards of care in both delivery modalities.

As we indicated at the outset, this is an exploratory investigation, and the naturalistic design introduced a number of limitations to our study. Because longitudinal data were not available for the sample examined, our investigation was limited to exploring differences between the telephone and in-person assessment conditions. The decision to work with data that emerged from the usual intake process at CBCS (with client consent), and thus not to disturb bereaved clients in the process of accessing support, meant forsaking a testretest study design, which may have been more robust.

Taken more broadly, it is important to acknowledge that it is the interest in providing not just care, but the right kind of care, for grieving people that creates the need for assessment in a bereavement intervention support program in the first place. Risk and symptom assessment enable a bereavement support system to operate a differentiated service model that accommodates clients with varying degrees of severity in their grief reactions or the character of their complaints. It also enables interviewers to discern between symptoms of grief and other underlying issues-such as trauma or substance abuse-which may require attention before a grief intervention can be of any help.

Finally, we would like to stress the need for caution when incorporating a telephone modality into an assessment system. Throughout the literature reviewed for this study, a common theme that emerged was researchers' consistent recommendations for using caution when making the decision to implement a telephone assessment modality for psychological and psychiatric services. This caution is especially important for practitioners and organizations considering the integration of telephone and in-person intake assessments into a single intake system-which is nearly always the case. In such situations, careful steps should be taken to account for differences introduced by a new assessment delivery method to ensure that clients assessed remotely will ultimately receive equivalent treatment as clients assessed in person. From the client's perspective, a well-run intake assessment process may help alleviate any trepidation associated with seeking help. For bereaved clients in 
particular, some of whom are seeking help for the first time in their lives, this is no small matter.

\section{References}

Baigent, M. F., Lloyd, C. J., Kavanagh, S. J., Ben-Tovim, D. I., Yellowlees, P. M., Kalucy, R. S., \& Bond, M. J. (1997). Telepsychiatry: "Tele" yes, but what about the "psychiatry"? Journal of Telemedicine and Telecare, 3(Suppl. 1), 3-5. doi:10.1258/1357633971930346

Brar, L. K., Brar, J. S., Deily, N. G., Wood, J. A., Reitz, P. M, Kupfer, D. J., \& Nimgaonkarb, V. L. (2002). Can clinical features of bipolar-I disorder be assessed reliably on the telephone? Journal of Affective Disorders, 71, 221-227. doi:10.1016/S0165-0327(01)00360-3

Cacciola, J. S., Alterman, A. I., Rutherford, M. J., McKay, J. R., \& May,D. J. (1999). Comparability of telephone and inperson structured clinical interview for DSM-III-R (SCID) diagnoses. Assessment, 6, 235-242. doi:10.1177/ 107319119900600304

Crawford, N. (2003). Knocking down access barriers. Monitor on Psychology, 34, 64.

Crippa, J. A. S., de Lima Osório, F., Del-Ben, C. M., Filho, A. S., da Silva Freitas, M. C., \& Loureiro, S. R. (2008). Comparability between telephone and face-to-face structured clinical interview for DSM-IV in assessing social anxiety disorder. Perspectives in Psychiatric Care, 44: 241-247. doi:10.1111/j.1744-6163.2008.00183.x

Cukor, P., Baer, L., Willis, B. S., Leahy, L., O’ Laughlen, J. O. H. N., Murphy, M., ... \& Martin, E. (1998). Use of videophones and low-cost standard telephone lines to provide a social presence in telepsychiatry. Telemedicine Journal, 4(4), 313-321. doi:10.1089/tmj.1.1998.4.313

Currier, J. M., Holland, J. M., \& Neimeyer, R. A. (2006). Sense-making, grief, and the experience of violent loss: Toward a mediational model. Death Studies, 30, 403-428. doi:10.1080/07481180600614351

Eaton, W. W., Anthony, J. C., Tepper, S., \& Dryman, A. (1992). Psychopathology and attirition in the epidemiologic catchment area surveys. American Journal of Epidemiology, 135(9), 1051-1059.

Freir, V., Kirkwood, K., Peck, D., Robertson, S., Scott-Lodge, L., \& Zeffert, S. (1999). Telemedicine for clinical psychology in the highlands of Scotland. Journal of Telehealth and Telemedicine, 5, 157-161. doi:10.1258/1357633991933567

García-Lizana, F., \& Muñoz-Mayorga, I. (2010). What about telepsychiatry? A systematic review. The Primary Care Companion to the Journal of Clinical Psychiatry, 12, e1-e5. doi:10.4088/PCC.09m00831whi

Hicks, L., Boles, K., Hudson, S., Kling, B., Tracy, J., Mitchell, J., \& Webb, W. (n.d.). Development of a telemedicine evaluation model. Retrieved from collab.nlm.nih.gov/ tutorialspublicationsandmaterials/.../6A-1.pdf

Hyler, S. E., Gangure, D. P., \& Batchelder, S. T. (2005). Can telepsychiatry replace in-person psychiatric assessments? A review and meta-analysis of comparison studies. CNS Spectrums, 10, 403-413.

Kavanagh, S. J., \& Yellowlees, P. M. (1995). Telemedicine: Clinical applications in mental health. Australian Family Physician, 24, 1242-1247.

Lee, S., Tsang, A., Mak, A., Lee, A., Lau, L., \& Ng, K. L. (2010). Concordance between telephone survey classification and face-to-face interview diagnosis of one-year major depressive episode in Hong Kong. Journal of Affective Disorders, 126, 155-160. doi:10.1016/j.jad.2010.03.024

Lyneham, H. J., \& Rapee, R. M. (2005). Agreement between telephone and in-person delivery of a structured interview for anxiety disorders in children. Journal of the American Academy of Child \& Adolescent Psychiatry, 44, 274-282. doi:10.1097/00004583-200503000-00012

Mozer, E., Franklin, B., \& Rose, J. (2008). Psychotherapeutic intervention by telephone. Journal of Clinical Interventions in Aging, 3, 391-396.

O’Reilly, R., Bishop, J., Maddox, K., Hutchinson, L., Fisman, M., \& Takhar, J. (2007). Is telepsychiatry equivalent to face-to-face psychiatry? Results from a randomized controlled equivalence trial. Psychiatric Services, 58, 836-843. doi:10.1176/appi.ps.58.6.836

Peñate, W. (2012). About the effectiveness of telehealth procedures in psychological treatments. International Journal of Clinical and Health Psychology, 12, 475-487.

Pinto-Meza, A., Serrano-Blanco, A., Peñarrubia, M. T., Blanco, E., \& Haro, J. M. (2005). Assessing depression in primary care with the PHQ-9: Can it be carried out over the telephone? Journal of General Internal Medicine, 20, 738-742. doi:10.1111/j.1525-1497.2005.0144.x

Prigerson, H. G., \& Jacobs, S. C. (2001). Diagnostic criteria for traumatic grief: A rationale, consensus criteria, and preliminary empirical test. In M. S. Stroebe, W. Stroebe, R. O. Hansson, \& H. Schut (Eds.), Handbook of bereavement research: Consequences, coping, and care (pp. 614-646). Washington, DC: American Psychological Association Press.

Prigerson, H. G., Maciejewski, P. K., Reynolds, C. F., III, Bierhals, A. J., Newsom, J. T., Fasiczka, A., ... Miller, M. (1995). Inventory of complicated grief: A scale to measure maladaptive symptoms of loss. Psychiatry Research, 59, 65-79. doi:10.1016/0165-1781(95)02757-2

Rohde, P., Lewinsohn, P. M., \& Seeley, J. R. (1997). Comparability of telephone and face-to-face interviews in assessing axis I and II disorders. American Journal of Psychiatry, 154, 1593-1598. doi:10.1176/ajp.154.11.1593

Schut, H., \& Stroebe, M. S. (2005). Interventions to enhance adaptation to bereavement. Journal of Palliative Medicine, 8(Suppl. 1), S140-S147. doi:10.1089/jpm.2005.8.s-140

Scottish Government. (2011). Scotland's capitals digital future: A strategy for Scotland. Retrieved from http://www.hie.co. uk/community-support/community-broadband-scotland/ news/broadband-help-for-rural-communities.html

Simon, G. E., Revicki, D., \& Von Korff, M. (1999). Telephone assessment of depression severity. Journal of Psychiatric Research, 27, 247-252. doi:10.1016/0022-3956(93)90035-Z

Spek, V., Nyklíček, I., Cujpers, P., \& Pop, V. (2008). Internet administration of the Edinburgh Depression Scale. Journal of Affective Disorders, 106, 301-305. doi:10.1016/j.jad.2007. 07.003

Stroebe, M. S., Folkman, S., Hansson, R. O., \& Schut, H. (2006). The prediction of bereavement outcome: Development of an integrative risk factor framework. Social Science \& Medicine, 63, 2440-2451. doi:10.1016/j.socscimed.2006.06.012

Zisook, S., Iglewicz, A., Avanzino, J., Maglione, J., Glorioso, D., Zetumer, S. \& Shear, M. K. (2014). Bereavement: Course, consequences, and care. Current psychiatry reports, 16, 1-10. doi:10.1007/s11920-014-0482-8 